Relations industrielles

Industrial Relations

\title{
Nature et raisons d'être des tribunaux du travail
}

\section{Émile Gosselin}

Volume 16, numéro 3, juillet 1961

URI : https://id.erudit.org/iderudit/1021775ar

DOI : https://doi.org/10.7202/1021775ar

Aller au sommaire du numéro

Éditeur(s)

Département des relations industrielles de l’Université Laval

ISSN

0034-379X (imprimé)

1703-8138 (numérique)

Découvrir la revue

Citer ce document

Gosselin, É. (1961). Nature et raisons d'être des tribunaux du travail. Relations industrielles / Industrial Relations, 16(3), 350-352.

https://doi.org/10.7202/1021775ar

Tous droits réservés @ C Département des relations industrielles de l’Université Laval, 1961
Ce document est protégé par la loi sur le droit d'auteur. L'utilisation des services d'Érudit (y compris la reproduction) est assujettie à sa politique d'utilisation que vous pouvez consulter en ligne.

https://apropos.erudit.org/fr/usagers/politique-dutilisation/ 
La première raison, c'est qu'il s'agit d'un problème sérieux, important, qui n'a jamais été résolu d'une façon systématique. On ne peut empêcher l'évolution sociale. Toute société se doit de posséder les mécanismes nécessaires à la sauvegarde de l'ordre public et des droits de ses ressortissants. Le problème du règlement des conflits de clroit en matière de relations du travail est crucial. Non seulement les droits des personnes et des institutions économico-sociales sont en jeu, mais souvent la paix et l'ordre de toute la société. Les conflits de droit en relations du travail, qu'ils soient individuels ou collectifs, existent. On ne peut les éviter. Il faut qu'ils soient réglés. Il est vrai que les tribunaux communs s'en occupent, que des commissions administratives sont graduellement amenées à exercer des fonctions judiciaires. Mais, est-ce suffisant? Est-ce satisfaisant? Est-ce que l'on doit continuer à se contenter de remèdes partiels sans tenir compte de solutions d'ensemble? Peut-ètre que oui, peut-ètre que non. Il importe pour le bien de tous que la lumière soit faite, que l'on sache où l'on va.

La seconde raison qui nous a poussé à étudier les tribunaux du travail, c'est que lcur création se trouve à être un des articles du programme électoral du parti qui vient de prendre le pouvoir (art. 30). Jusqu'à quel point faut-il accorder du sérieux à un programme électoral? L'histoire nous a appris à garder beaucoup de réserve là-dessus et nous laissons à chacun le soin de porter son propre jugement. Mais il est bon de se préparer à toute éventualité. Ce n'est que simple prudence. Dans ce cas-ci, c'est un motif supplémentaire pour s'empresser de déblayer le terrain, de faire une étude objective avant que la passion, les intérêts, le parti-pris n'entrent en scène.

Le Département des relations industrielles de Laval n'est pas à la solde de qui que ce soit: gouvernement, syndicats ouvriers ou employeurs. Il est au service du public. Son rôle n'est pas d'apporter des solutions toutes faites, de dicter des lignes de conduite, mais bien de poser les problèmes, de stimuler la pensée, d'établir toutes les hypothèses, laissant ensuite aux intéressés le soin de prendre les décisions.

Le congrès des relations industrielles de Laval réunit tous ceux qui dans cette province s'occupent des relations du travail tant sur le plan académique que sur le plan de l'action. Il est une occasion pour tous de se rencontrer sur un terrain neutre et de discuter leurs problèmes dans des perspectives d'objectivité. C'est ce que nous voulons faire avec celui des tribunaux du travail.

\section{Nature et raisons d'être des tribunaux du travail}

\section{EMILE Gosselin}

L'accélération du rythme de l'industrialisation a vu s'accroître la fréquence et l'étendue de l'intervention gouvernementale. Il en est résulté une abondante législation sociale accompagnée d'une forte délégation de pouvoirs réglementaires et quasi-judiciaires à de nombreux organismes publics ou quasi-publics. L'Etat a non seulement obligé les individus et les groupes à de nouvelles façons d'agir, mais en élaborant un droit social différent de l'ordre juridique antérieur, il a consacré des droits nouveaux et, dans une certaine mesure, reformulé une nouvelle échelle de valeurs juridiques. 
Le droit du travail gouverne le travail subordonné, c'est-à-dire l'ensemble des rapports juridiques qui naissent à l'occasion de l'accomplissement d'un travail subordonné. Ce droit couvre à la fois les rapports individuels et collectifs de travail, et englobe également des lois de protection au travail ainsi qu'une série de mesures de sécurité sociale impliquant le travailleur en tant quaccomplissant un travail subordonné.

La masse de législations et de réglementations, ainsi que la profusion des divers organismes administratifs chargés d'administrer ces lois nouvelles ou d'en sanctionner l'application, crée beaucoup de confusion et souvent du malaise chez ceux qui cherchent à faire valoir leurs droits dans le secteur du travail. En premier lieu, les organismes se sont ajoutés aux organismes, chacun possédant une juridiction qui lui est propre, des pouvoirs dont l'étendue varie, des règles de procédure plus ou moins précises ou stables. En outre, le mode de recrutement du personnel requis par ces organismes varie selon le cas. Enfin, ce qu'il en coûte pour faire valoir ses droits et les délais qu'il faut encourir selon que l'on s'adresse à une juridiction plutôt qu'à une autre constitue des éléments forts variables et de la plus haute importance pour les économiquement faibles.

En dépit du fait que l'on ait dessaisi les tribunaux ordinaires de pouvoirs ou de rôles qu'ils jouaient traditionnellement dans l'administration de la chose publique, de nombreuses critiques continuent de s'élever à leur endroit. De plus, les organismes administratifs nouveaux chargés par le législateur de l'administration et de la mise en force des lois du travail, font également l'objet de nombreux griefs dans l'exercice de leurs pouvoirs judiciaires.

L'un des reproches que l'on formule habituellement à l'endroit des tribunaux ordinaires constitue un jugement historique au sujet de leur place dans l'ensemble du système juridique et judiciaire. Ces tribunaux sont apparus à une époque où l'on croyait que l'intérêt public serait le mieux servi, si on laissait aux individus eux-mêmes le soin de procéder devant les tribunaux. L'appareil judiciaire, en un tel cas, joue un rôle passif et n'agit que sur demande de la partie lésée. Un tel système, conçu surtout en vue de protéger des droits individuels et constituant un monopole de la fonction juridictionnelle confiée aux tribunaux ordinaires, était concevable à une époque caractérisée par la non-intervention gouvernementale. Lorsque le législateur passait une loi, elle était fort détaillée et elle énumérait exactement les droits et les obligations des individus. L'administration de la justice en était facilitée dès lors. En conséquence, l'administrateur public jouait un rôle minime sur le plan réglementaire et possédait peu ou pas de pouvoirs judiciaires. Mais en déléguant des pouvoirs réglementaires à des organismes administratifs, le législateur leur a également conféré des pouvoirs quasi-judiciaires en vue d'accélérer la mise en force des lois et des règlements.

L'un des griefs que l'on soulève à l'égard de l'administration de nos lois ouvrières résulte du fait qu'il faut encore trop souvent recourir aux tribunaux ordinaires en vue de donner des sanctions efficaces aux lois du travail ou aux accords passés en vertu de ces lois. Il en résulte des délais trop considérables, conséquence de la surcharge des tribunaux ordinaires, des procédures compliquées et souvent de mesures dilatoires. En outre du coût parfois prohibitif des causes civiles, la partie lésée se rend compte souvent que le remède apporté par le tribunal civil est illusoire. 
Par contre, des griefs s'élèvent également au sujet des organismes administratifs pourvus de pouvoirs quasi-judiciaires. Ou bien l'on invoquera que l'absence d'appel de leurs décisions va à l'encontre d'une protection équitable des droits de l'individu, ou encore que l'absence de procédures définies et stables rend difficile l'exercice des droits et la sanction des obligations prévues par les lois du travail. On s'élève, en nombre de milieux, contre le fait que l'on recourt trop souvent aux tribunaux ordinaires pour empêcher les organismes administratifs de jouer pleinement leurs rôles. Selon l'optique que l'on a du rôle et des pouvoirs de tels organismes, l'on invoquera quils possèdent trop de pouvoirs en certains cas, pas assez en d'autres. Enfin, l'on invoque qu'il y a trop d'organismes possédant des pouvoirs quasi-judiciaires et qu'une grande confusion règne du fait de la multiplicité des organismes auxquels il faut recourir et des règles changeantes de la procédure, car chaque organisme possède les siennes.

Ainsi, en nombre de milieux, réclame-t-on la création de tribunaux du travail, car, en dessaisissant les organismes administratifs de leurs fonctions judiciaires, on les amènerait à jouer surtout des rôles proprement réglementaires et proprement administratifs.

Il ne saurait être question de confier aux tribunaux administratifs dans le domaine du droit du travail le règlement des conflits d'intérêt. Ce domaine n'appartient à aucun tribunal, quel qu'il soit. Mais peut-on confier le règlement de tous les conflits de droit du travail à de tels tribunaux?

Avant de répondre à cette question, il faut s'interroger sur le rôle de la fonction juridictionnelle et des organismes qui en sont revêtus par rapport à chaque palier de conflit. Certains conflits résultent de l'application ou de l'interprétation de lois ou de règles sur lesquelles les organismes administratifs n'ont aucun contrôle, sauf parfois de suggérer des modifications au législateur. En d'autres cas, il s'agit de conflits de droits résultant de l'application de la réglementation tombant sous la juridiction des organismes administratifs. Certains cas impliquent strictement des individus, par exemple dans le cas de l'application de la Loi des accidents du travail. En d'autres cas, des droits collectifs sont en cause. Enfin certaines règles de droit résultent d'accords collectifs. Notons bien ceci: avant de diviser la fonction juridictionnelle des organismes administratifs, il faut bien voir l'importance de cette fonction dans la dynamique même de l'administration publique moderne. C'est à de telles questions que les différents conférenciers, lors du 16ème Congrès des Relations Industrielles, devront apporter, à tout le moins, des débuts de réponse.

\section{Expériences étrangères}

\section{René Mankiewicz}

Au moment où se pose au Québec la question de savoir s'il convient de créer des tribunaux du travail et quelles seraient leurs fonctions, il n'est pas sans intérêt d'examiner les expériences faites par d'autres pays dans ce domaine. On se rend ainsi compte de l'ampleur du problème et de la diversité des solutions adoptées à travers le monde.

En effet la création de tribunaux de travail soulève des problèmes complexes, et cela à cause de la diversité même des conflits de travail. Certains différends, par exemple ceux qui surgissent lors de la conclusion d'une convention collective 